\title{
Tuberculosis Otitis Media with Disseminated Tuberculosis in a Pregnant Female
}

\author{
Jennifer Jubulis ${ }^{1}$, Stephen Riedel ${ }^{2}$, Nicole Parrish ${ }^{2}$ \\ ${ }^{1}$ Division of Pediatric Infectious Disease, Maine Medical Center/Tufts University School of Medicine, \\ Portland, USA \\ ${ }^{2}$ Department of Microbiology, Johns Hopkins Hospital, Baltimore, USA \\ Email: JUBULJ@mmc.org
}

Received 9 February 2014; revised 14 March 2014; accepted 20 March 2014

Copyright (C) 2014 by authors and Scientific Research Publishing Inc.

This work is licensed under the Creative Commons Attribution International License (CC BY).

http://creativecommons.org/licenses/by/4.0/

(c) (i) Open Access

\begin{abstract}
Background: Tuberculosis remains a public health problem worldwide, and tuberculosis in pregnancy in particular can have effects on both mother and infant. Findings: We report a case of a 22 year-old female presenting in preterm labor at 32 weeks gestation. Her pregnancy had been complicated by hearing loss and weight loss, and at the time of delivery, she was diagnosed with tuberculous otitis media and disseminated tuberculosis. Tuberculous otitis media is a rare form of tuberculosis disease, and in this case, had consequences for mother and infant. Conclusions: Importance of suspicion for tuberculosis disease in patients presenting with atypical symptoms but epidemiologic history is emphasized.
\end{abstract}

\section{Keywords}

Tuberculosis, Tuberculous Otitis Media, Neonate, Pediatric Tuberculosis

\section{Introduction}

Tuberculosis (TB) is a common infectious disease worldwide. Approximately nine million new cases are registered annually, with two thirds of these cases occurring in patients between 15 and 64 years of age [1]. This is of particular concern as this age range encompasses women of childbearing age. Pregnant women may be particularly at risk for development of active TB disease, due to decreased cellular immunity during their time of pregnancy [1]. Because of decreased immunity and atypical extrapulmonary manifestations of the disease, the diagnosis of TB may be difficult in pregnant women. Overall, 15\% of TB cases are extrapulmonary; however, one study 
found that as many as $52 \%$ of pregnant women with TB have extrapulmonary manifestations [1]. Also important to consider is the fact that in pregnant women, two patients are at risk. While the exact risk of transmission to the fetus or newborn is unknown, a prompt diagnosis is essential to implement effective and timely antimicrobial treatment. This case report highlights dilemmas inherent in tuberculosis diagnosis, and the importance of awareness of rare forms of the disease in patients with an epidemiologic background consistent with TB disease.

\section{Case Report}

A 22-year-old Hispanic female with a diagnosis of preterm prolonged rupture of membranes and spontaneous vaginal delivery at 32 and 1/7 weeks was seen by the infectious disease service at our institution due to chronic otitis media. One year prior to the current admission, she presented with mild hearing loss in her right ear, followed by purulent drainage from her left ear, odynophagia, and gradually progressive hearing loss on the same side over a 4 month period. At that time, she was seen by an otolaryngologist and was treated with amoxicillinclavulanate and steroids. Her symptoms initially improved over the next several weeks but recurred one month later. A follow-up ENT examination, including a fiberoptic exam of the upper airways, demonstrated "significant supraglottitis with ball-like effacement of the epiglottis, false vocal cords, and arytenoids". The patient was admitted for treatment of acute otitis media and supraglottitis. Treatment with clindamycin, ceftriaxone, and dexamethasone was begun, and the patient was discharged to home after 4 days to complete 14 days of clindamycin.

The patient was then admitted 2 weeks later with preterm prolonged rupture of membranes. After 11 hours of rupture, she delivered a $1500 \mathrm{~g}$ infant appropriate for gestational age. On the day of delivery, the mother was noted to have a fever of $39.4^{\circ} \mathrm{C}$ and a white blood cell count of 12,520 cells $/ \mathrm{mm}^{3}$. She was also tachycardic and had fundal tenderness. Based on her symptoms, she was empirically treated with ampicillin, gentamicin, and clindamycin for 48 hours for presumed chorioamnionitis, and defervesced. However, she then developed a fever to $38.5^{\circ} \mathrm{C}$. A chest radiograph showed diffuse bilateral infiltrates, and she was started on ceftriaxone and azithromycin for presumed pneumonia.

Upon review of systems at that time, the patient further described dysuria of 6 months duration, shortness of breath, inability to lie flat due to increased respiratory secretions, and productive cough. She also complained of diffuse myalgias in her arms, back, and legs, with no joint involvement. She reported having intermittent night sweats and fever for the past 7 months. The patient had unintentionally lost $27 \mathrm{lbs}$ during her pregnancy.

The patient was born in Ecuador and had moved to the United States approximately one year prior to her current presentation to our hospital. In Ecuador, she had completed all immunizations and had received a BCG vaccine as a child. It is unclear whether she had ever had a tuberculin skin test (TST) placed.

Upon examination by our infectious diseases service, the patient was febrile to $38.7^{\circ} \mathrm{C}$ and tachycardic. Respirations were $18 / \mathrm{min}$ and oxygen saturation was $97 \%$ on room air. Blood and pus were present behind the tympanic membranes bilaterally, with some drainage from the left ear. She had some tender submandibular lymphadenopathy.

Laboratory studies showed normal electrolytes and creatinine. A complete blood count showed a total white cell count of 12,520 cells $/ \mathrm{mm}^{3}$ with $73 \%$ neutrophils and $19 \%$ bands. The auramine-rhodamine stained smear of the expectorated sputum sample was positive for acid fast bacilli (AFB), as was drainage from the ear. The HIV screen was negative. Based on these findings, the patient was started on a 4-drug regimen consisting of isoniazid, rifampin, pyrazinamide, and ethambutol. Despite a negative chest radiograph after delivery, the infant was started prophylactically on isoniazid and rifampin. In addition, blood cultures were obtained on the infant, but remained negative and the infant remained asymptomatic. As a precautionary measure, the infant and mother remained separated at/after delivery and during hospitalization, until the mother had three negative sputum smears.

\section{Discussion}

Tuberculous otitis media (TOM) accounts for only a small percentage $(0.9 \%)$ of patients with chronic otitis [2]. This illness is also difficult to diagnose due to multiple factors: low prevalence leading to a low index of suspicion, clinical signs that vary from the classic description, infrequent association with other systemic involvement, and false negative cultures due to the fastidious nature of Mycobacterium tuberculosis [3]. However, failure to identify and treat this illness can lead to permanent hearing loss [3] [4]. Furthermore, failure to diagnose TB in a 
pregnant patient such as the one described here, may complicate the delivery and early infant life [1] [5]-[7].

The pathogenesis of TOM has been the subject of debate. Three mechanisms are proposed; spread to the middle ear from the Eustacian tube, hematogenous spread from another focus, and direct implantation through the external auditory canal and tympanic membrane perforation [2] [4]. It is highly likely that our patient developed $\mathrm{TOM}$ as a result of a disseminated pulmonary infection, based on chest imaging and sputum culture results.

Medical management of TOM is the same as for pulmonary tuberculosis [4]. Outcome is typically excellent if therapy is initiated early in the disease course; however, delays in diagnosis lead to complications such as facial paralysis and central nervous system involvement [4]. Surgery may be indicated in patients with complicated TOM, including those patients with abscesses, fistula, facial palsy, or intracranial extension of infection [4]. In a study examining 16 ears of 14 patients with TOM treated either medically or medically and surgically, dry ears were obtained with chemotherapy alone in all patients but one; however, normalized tympanic membranes were found in $50 \%$ of the chemotherapy group and $75 \%$ of the surgery group. Duration of otorrhea in the groups was not statistically different. The study did find that the frequency of hearing improvement was higher in the surgical group [4]. Due to disseminated disease in this patient, she was managed with 4-drug, directly observed therapy (DOTS) and recovered. However, residual hearing loss has continued.

This case demonstrates how the diagnosis of TB, including rare forms such as TOM can have far reaching consequences for both pregnant women and their infants. TB disease is difficult to diagnose in young children, and young children, once infected, are at high risk for development of disseminated TB including TB meningitis. Guidelines exist for management of infants born to women with pulmonary disease and consist of 6 months of isoniazid prophylactic therapy [7]. However, such guidelines are lacking for infants born to mothers with extra-pulmonary disease $[5]$.

Maternal TB places neonates at risk of preterm delivery and/or growth delay [5]-[7]. Studies from India report that even if infants born to mothers with tuberculosis remain uninfected, they are more likely to be of low birth weight than healthy controls ( $34.2 \%$ vs $6.3 \%$ ) and have an increased risk of being born prematurely ( $22.8 \%$ vs $11.1 \%$, RR 2.1, P < 0.01) [1]. In a study from Mexico, the authors found that pregnant women with tuberculosis experienced a 9-fold increased risk of preterm labor when compared to women without tuberculosis [1]. In the current case, the infant was born at 32 weeks gestation, possibly as a result of severe maternal tuberculosis disease. This infant received a full course of prophylactic therapy, supervised by the Health Department, and was then lost to follow up.

\section{Conclusion}

This case of TOM in this 22-year-old woman illustrates the difficulties in making an accurate and timely diagnosis of TB, particularly in cases of pregnancy, which may lead to long standing complications. TOM should be considered that in cases of chronic otitis media in patients from a TB endemic area, and pregnant patients should be followed, as should their babies, to ensure proper treatment.

\section{Acknowledgements}

The authors wish to acknowledge Karen Carroll and Sanjay Jain for their editorial support for this paper.

\section{References}

[1] Adhikari, M. (2009) Tuberculosis and Tuberculosis/HIV Co-Infection in Pregnancy. Seminars in Fetal and Neonatal Medicine, 14, 234-240.

[2] Richardus, R.A., Jansen, J.C., Steens, S.C. and Arend, S.M. (2011) Two Immigrants with Tuberculosis of the Ear, Nose, and Throat Region with Skull Base and Cranial Nerve Involvement. Case Reports in Medicine, 2011, Article ID: 675807. http://dx.doi.org/10.1155/2011/675807

[3] Kim, D.K., Park, S.N., Park, K.H. and Yeo, S.W. (2014) A Case of Direct Intracranial Extension of Tuberculous Otitis Media. Ear, Nose \& Throat Journal, 2, 68-74.

[4] Kwon, M., Choi, S. and Chung, J. (2010) Roles of an Anti-Tuberculosis Medication and Surgery in Patients with Tuberculous Otitis Media. ActaOto-Laryngologica, 130, 679-686. http://dx.doi.org/10.3109/00016480903311260

[5] De Schutter, I., Schepers, K.,Singh, M., Mascart, F. and Malfroot, A. (2010) Latent Tuberculosis in a Newborn: Diagnostic Challenges. European Journal of Pediatrics, 169, 1155-1158. http://dx.doi.org/10.1007/s00431-010-1177-8 
[6] Getahun, H., Sculier, D., Sismanidis, C., Grzemska, M. and Raviglione, M. (2012) Preention, Diagnosis, and Treatment of Tuberculosis in Children and Mothers: Evidence for Action for Maternal, Neonatal, and Child Health Services. JID, 2005, S216-S227. http://dx.doi.org/1-.1093/infdis/jis.009

[7] WHO (2006) Guidance for National Tuberculosis Programmes on the Management of Tuberculosis in Children. http://www.who.int/maternal_child_adolescent/documents/htm tb_2006 371/en/ 